\title{
Development of a resonant scanner to improve the imaging rate of astigmatic optical profilometers
}

\author{
Liao, Hsien-Shun; Cheng, Shih-Han; Hwu, En Te
}

Published in:

IEEE/ASME Transactions on Mechatronics

Link to article, DOI:

10.1109/TMECH.2020.3011465

Publication date:

2021

Document Version

Peer reviewed version

Link back to DTU Orbit

Citation (APA):

Liao, H-S., Cheng, S-H., \& Hwu, E. T. (2021). Development of a resonant scanner to improve the imaging rate of astigmatic optical profilometers. IEEE/ASME Transactions on Mechatronics, 26(2), 1172-1177.

https://doi.org/10.1109/TMECH.2020.3011465

\section{General rights}

Copyright and moral rights for the publications made accessible in the public portal are retained by the authors and/or other copyright owners and it is a condition of accessing publications that users recognise and abide by the legal requirements associated with these rights.

- Users may download and print one copy of any publication from the public portal for the purpose of private study or research.

- You may not further distribute the material or use it for any profit-making activity or commercial gain

- You may freely distribute the URL identifying the publication in the public portal

If you believe that this document breaches copyright please contact us providing details, and we will remove access to the work immediately and investigate your claim. 


\title{
Development of a resonant scanner to improve the imaging rate of astigmatic optical profilometers
}

\author{
Hsien-Shun Liao, Shih-Han Cheng, and En-Te Hwu
}

\begin{abstract}
An astigmatic optical profilometer provides numerous advantages such as compact size, low cost, and high resolution. In 2018, a $\mathrm{z}$-axis modulation mode of the astigmatic optical profilometer was developed to realize quantitative height measurement for a surface comprising complex materials. However, the current imaging rate of the z-axis modulation mode is time consuming. In this work, a resonant scanner was proposed to execute a high-speed $z$-axis scanning motion. By inducing the resonant mode, the resonant scanner enabled both a large trave range of over $87 \mu \mathrm{m}$ and high oscillation frequency of $1.576 \mathrm{kHz}$. Additionally, a data analysis process was proposed to calibrate the nonlinear movement of the resonant scanner. Experimental results demonstrated that the developed optical profilometer successfully captured the quantitative height and reflectivity of the complex material comprising chrome patterns on a glass substrate. Furthermore, an imaging rate of $256 \mathrm{~s} /$ frame was achieved, which was approximately 50 times faster than that of a previous system employing a commercial flexure-guided scanner.
\end{abstract}

Index Terms-High-speed scanning, optical profilometer, piezoelectric actuator, resonant scanner.

\section{INTRODUCTION}

$\mathrm{O}$ PTICAL profilometer is widely used for measuring the surface morphology of various materials such as optical components and biomaterials [1]-[3]. Different optical methods, such as focus detection, interferometry, and the astigmatic method, have been employed to realize optical profilometers [4]-[7]. On adopting a commercial digital video disc (DVD) pickup head, an astigmatic optical profilometer provides advantages including compact size, low cost, high resolution, and easy alignment [8]-[10]. Moreover, the astigmatic optical profilometer can be converted into a stylus profilometer by adding a sharp probe, which provides profile measurements at nanometer resolution [11]. In the astigmatic method, a laser is focused on a sample surface, and the reflective laser is detected using a photo detector integrated chip (PDIC). An astigmatic lens in an optical path changes the

H.-S. Liao and S.-H. Cheng are with the Department of Mechanical Engineering, National Taiwan University, Taipei 10617, Taiwan (e-mail: liaohs@ntu.edu.tw; r07522638@ntu.edu.tw).

En-Te Hwu is with the Department of Micro- and Nanotechnology, Technical University of Denmark, Lyngby 2800, Denmark (e-mail: etehw@dtu.dk). shape of the laser spot on the PDIC according to the change in distance between the focal laser and sample. The relation between a focus error signal $V_{F E S}$ from the PDIC and sample position is called the $\mathrm{S}$-curve. In the linear region of the $\mathrm{S}$-curve, $V_{F E S}$ is proportional to the sample position and therefore can represent the sample morphology by scanning the sample surface. However, the magnitude of $V_{F E S}$ is affected by the surface reflectivity. Therefore, the quantitative height image is not available on a surface comprising complex materials. To overcome this limitation, we previously proposed a z-axis modulation method for obtaining a quantitative height image through the addition of a sample scanning movement in the $\mathrm{z}$-axis direction [12]. However, three-dimensional scanning is time consuming. The current imaging rate is $3.5 \mathrm{~h}$ for capturing one image. The current speed is limited by the bandwidth of the scanner.

Piezoelectric actuators exhibit the advantages of high precision, compact size, and easy control [13], [14], which have been applied in various fields such as precision machining and atomic force microscopy (AFM) [15], [16]. To realize a high-speed AFM for observing dynamic phenomena at nanoscale, various flexure-guided piezoelectric scanners have been proposed [17], [18]. The bandwidth of piezoelectric scanners can be improved by increasing the stiffness of the actuator structure. However, a trade-off between the stiffness and travel range of the flexure-guided actuator exists [19] , [20]. For instance, the resonant frequency of the flexure-guided scanner with a travel range of $100 \mu \mathrm{m}$ is approximately $500 \mathrm{~Hz}$. A triangular waveform is commonly adopted to drive the flexure-guided scanners. Due to the harmonics of the triangular waveform, the practical driving frequency is usually lower than one-tenth of the resonant frequency of the scanner to avoid the resonances. By contrast, resonant scanners overcome this problem by driving at their resonant frequency. Humphris et al. used a tuning fork with a resonant frequency of $20 \mathrm{kHz}$ to carry a sample for high-speed AFM scanning. However, the sample size is extremely limited due to the size of the tuning fork [21]. To overcome this issue, different designs such as the quartz bar and the piezoelectric bimorph-based scanner were proposed [22]-[25]. The quartz bar scanner developed by Zhao et al. achieved a travel range of $37.5 \mu \mathrm{m}$ at its resonant frequency of $772 \mathrm{~Hz}$ [22]. Wang et al. further demonstrated that the travel range and the resonant frequency can be designed through modifying the length of the quartz bar. Through using a long 
quartz bar with a size of $4 \mathrm{~mm} \times 5.5 \mathrm{~mm} \times 66 \mathrm{~mm}$ (thickness $\times$ height $\times$ length), a large travel range of $170 \mu \mathrm{m}$ was achieved at its resonant frequency of $830 \mathrm{~Hz}$ [25]. Both the quartz bar and bimorph-based scanners utilize the first resonant mode of the cantilever beam structure to magnify the travel range. The cantilever structure is simple, and its resonant frequency can be evaluated easily. However, the movement at the free-end of the cantilever includes both translational and angular displacements. The angular displacement is undesired in the astigmatic optical profilometer, because the angular tilt may cause a distortion of the S-curve.

In this work, we designed a resonant scanner to achieve the requirements of both high speed and large scanning range for the z-axis scanning movement. Through using a double-end fixed beam structure, the designed resonant scanner can perform a pure vertical movement. The main drawbacks of the resonant scanners were an unchangeable scanning rate and a nonlinear scanning trajectory. The resonant frequency of the proposed resonant scanner was determined by designing the dimensions of the flexural structure. The developed resonant scanner exhibited a resonant frequency of $1.576 \mathrm{kHz}$ and a full scan range of $>87 \mu \mathrm{m}$. Moreover, a data analysis process was proposed for calculating the nonlinear displacement of the scanner. The resonant scanner was integrated into a homemade

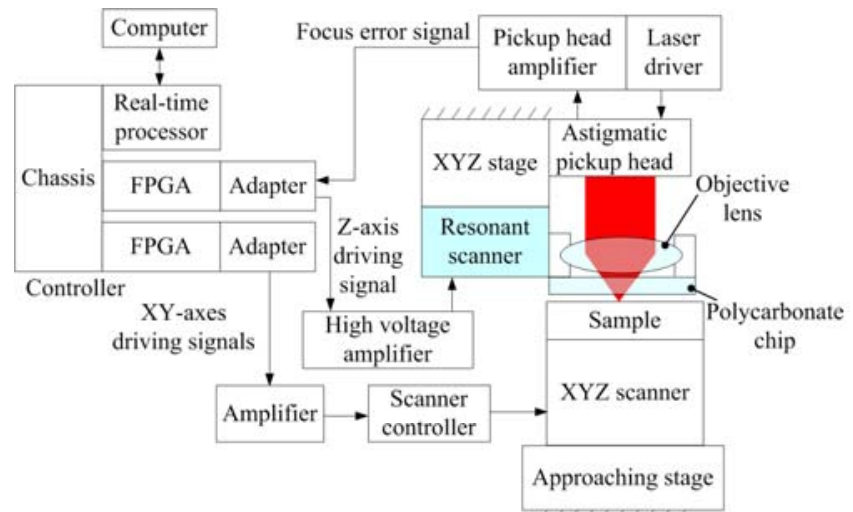

(a)

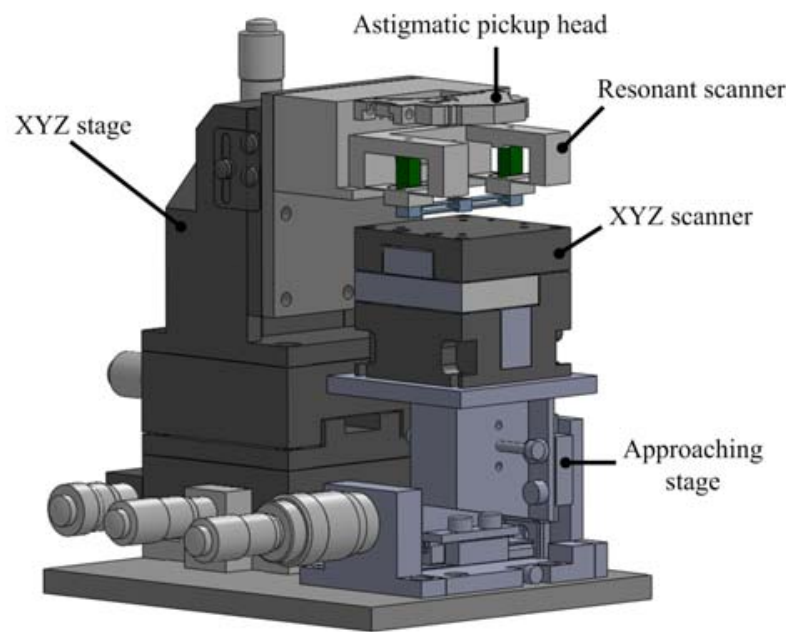

(b)

Fig. 1. (a) System configuration and (b) mechanical design of the astigmatic optical profilometer. astigmatic optical profilometer, and the developed system successfully captured the quantitative height and reflectivity images of a glass substrate with a chrome coating pattern. The experiment was executed at a scan rate of 1 line/s ( $256 \mathrm{~s} /$ frame), which was approximately 50 times faster than that of a previous system (3.5 h/frame) [12].

\section{INSTRUMENTATION}

In this section, the system configuration of the homemade astigmatic optical profilometer and the design of the resonant scanner are described. To obtain the relation between $V_{F E S}$ and scanner displacement, a data analysis process was also proposed.

\section{A. Astigmatic optical profilometer}

The system configuration and mechanical design of the homemade astigmatic optical profilometer are presented in Fig. 1(a) and (b), respectively. An astigmatic pickup head (TOP1100s, TopRay Technologies) was employed to measure the surface height and reflectivity. To achieve fast z-axis modulation, a novel resonant scanner was developed to carry the objective lens of the pickup head for the vertical $\mathrm{z}$-axis scanning. Both the resonant scanner and pickup head were installed on an XYZ stage (XC2-60SMW and ZA-60MBW, TWIN NINES) to adjust the measuring position on the sample. A customized amplifier (high-speed Topray driver v122, Strømlinet Nano) was used to drive a laser diode and to generate the focus error signal $V_{F E S}$ from the PDIC in the pickup head. The focus error signal was acquired using a programmable controller (PXIe system, National Instruments), consisting of a chassis (PXIe-1062Q), a real-time controller (PXIe-8840), and two field-programmable gate array (FPGA) modules (PXIe-7961R) with the adaptors (NI5781). One FPGA module was responsible for capturing the focus error signal at a sampling rate of $40 \mathrm{MHz}$ and generating a sinusoidal signal for $\mathrm{Z}$-axis modulation. The sinusoidal signal $(-1$ to $1 \mathrm{~V})$ was amplified using a high voltage amplifier (PD200, PiezoDrive) with a gain of $75(0-150 \mathrm{~V})$ to drive the resonant scanner. Another FPGA module was employed to generate $x y$-axis signals to control the scanning movement of a closed-loop XYZ piezoelectrical scanner (P-611.3S NanoCube, Physik Instrumente). The closed-loop XYZ scanner adopted a flexure-guided design, and its resonant frequencies in the $\mathrm{X}, \mathrm{Y}$, and $\mathrm{Z}$ directions were 350,220 , and $250 \mathrm{~Hz}$, respectively. A homemade amplifier was used to magnify the scanning signals $(-1$ to $1 \mathrm{~V})$ from the FPGA module to fit the input voltage range $(-2$ to $12 \mathrm{~V})$ of the scanner controller (E-664.S3 piezo controller, Physik Instrumente), which adopted a proportional-integral (PI) control circuit to achieve accurate positioning. The vertical movement of an XYZ scanner was only used in the initial displacement calibration of the resonant scanner and was kept stationary during the scanning measurement. An approaching stage was used to transfer the sample to the measuring range of the pickup head. 


\section{B. Resonant scanner}

Figure 2(a) illustrates the mechanical design of the resonant scanner. Two piezoelectric stack actuators (AE0505D08DF, Tokin) were used to oscillate the resonance of the flexural structure. Piezoelectric actuators exhibited an unload displacement of $9.1 \pm 1.5 \mu \mathrm{m}$ at a driving voltage of $150 \mathrm{~V}$ and a typical resonant frequency of approximately $138 \mathrm{kHz}$. A stainless steel frame clamped the piezoelectric actuators to provide a preload and connected the flexural structure. The flexural structure was designed for oscillating at the first resonant mode to achieve the high-speed $\mathrm{z}$-axis scanning motion. The flexural structure was fixed at both ends, which can provide a pure vertical displacement at its midpoint. The parallel beams of the flexural structure were designed to reduce the torsional deformation. The objective lens was glued in the middle cavity of the flexural structure. A polycarbonate chip from a DVD was placed under the objective lens to enhance $V_{F E S}$. The resonant frequency of the flexural structure can be designed by adjusting the dimension and material. Figure 2(b) illustrates the dimension of the flexural structure, and the structure was fabricated using stainless steel.

A finite element analysis with the COMSOL Multiphysics software was used to evaluate resonant modes and corresponding frequencies. For the structure steel, the build-in material properties $($ density $=7850 \mathrm{~kg} / \mathrm{m} 3$, Young's modulus $=$ $200 \mathrm{GPa}$, and Poisson's ratio $=0.33$ ) were applied. The mass of the objective lens and the polycarbonate chip was neglected in the simulation. Figure 3(a)-(c) illustrates the first three resonant modes at the resonant frequencies of 1.94, 9.45, and $9.95 \mathrm{kHz}$, respectively. The resonant frequencies of the second and third modes were much higher than those of the first mode. Therefore, the effect of high-order resonant modes can be neglected during scanning.

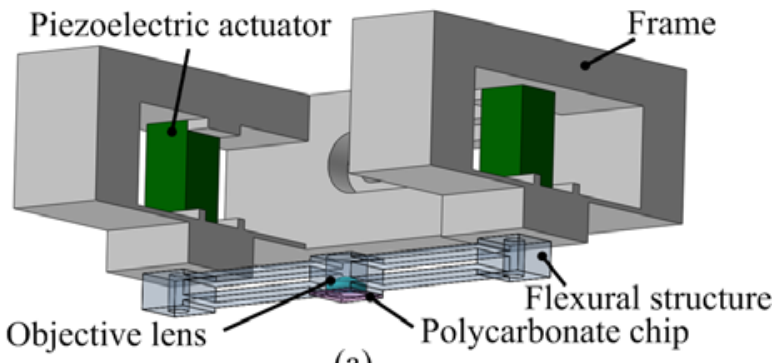

(a)
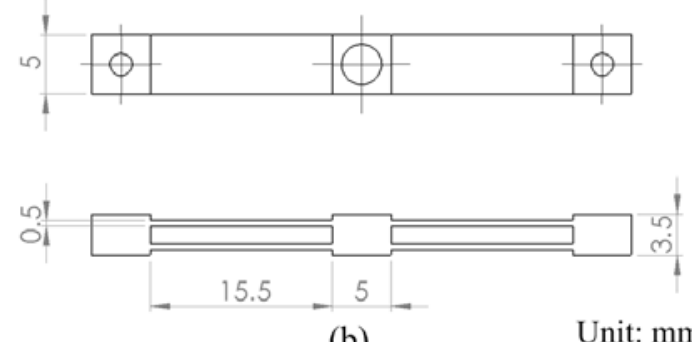

(b)

Unit: mm

Fig. 2. (a) Mechanical design of the resonant scanner. (b) Dimension of the flexural structure.

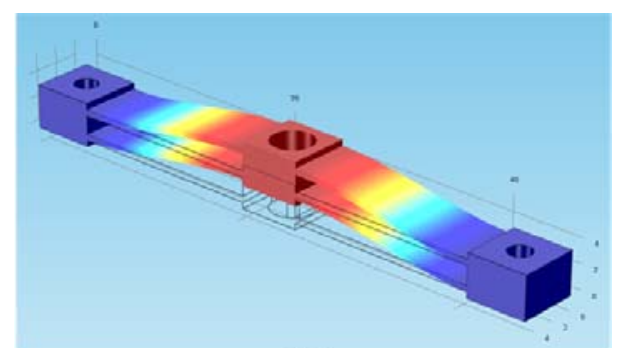

(a)

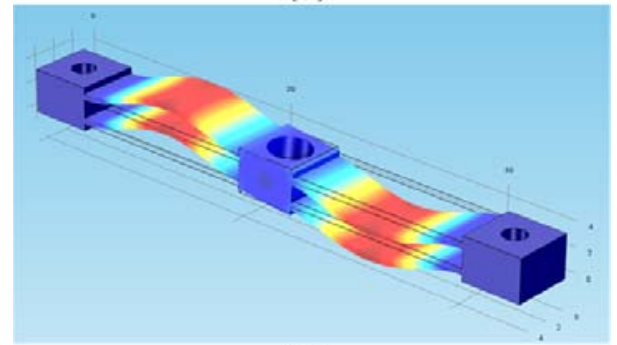

(b)

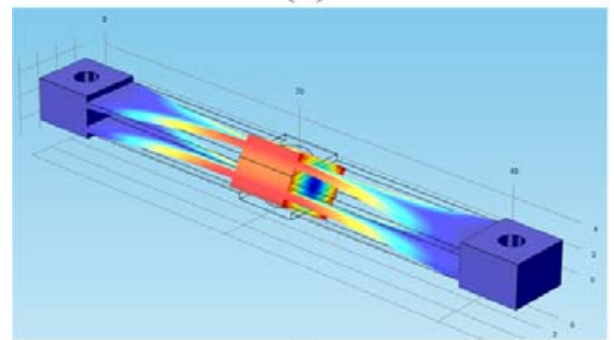

(c)

Fig. 3. Finite element analysis: (a) first, (b) second, and (c) third resonant modes of the flexural structure.

\section{Data analysis}

During scanning, the resonant scanner was oscillated at its resonant frequency for executing the $\mathrm{Z}$-axis scanning movement, and at each pixel, S-curves were recorded and analyzed to obtain the surface height and reflectivity. An additional analysis process was required due to the sinusoidal movement of the resonant scanner. The driving signal generated using the FPGA module and the actual displacement of the resonant scanner are illustrated in Fig. 4(a). A phase lag between the driving signal and scanner displacement existed, which varied with the driving frequency around the resonant frequency. The unknown phase lag was obtained through identifying the turning points of the scanner displacement in the S-curve measurement as illustrated in Fig. 4(b). The S-curves measured during the upward and downward movements of the scanner were opposite to each other, and the mirror lines indicated the upper and lower turning points of the scanner movement. The time points of the turning points were compared with the driving signal to evaluate the phase lag. Moreover, to obtain a plot of the focus error signal $V_{F E S}$ versus displacement, the amplitude of the scanner displacement must also be calibrated. For calibrating the displacement amplitude, the well-calibrated closed-loop XYZ scanner was used to adjust the Z-axis position of the sample to shift the S-curve along the time axis. The focal position of the pickup head was adjusted to the upper and lower turning points, respectively. The 
corresponding z-axis positions of the closed-loop XYZ scanner at the two turning points were recorded, and the distance between the two turning position was twice the oscillation amplitude. After calibrating the phase lag and the displacement amplitude, the displacement curve of the scanner in Fig. 4(a) can be obtained, then the graph of $V_{F E S}$ versus displacement can be plotted as illustrated in Fig. 4(c). To calculate the surface height, the focal position was calculated through linear curve fitting in the linear region of the S-curve. Moreover, the slope $\Delta V_{F E S} / \Delta Z$ of the linear region was obtained, which represented the surface reflectivity. S-curves were analyzed at each pixel by repeating this process to obtain the height and reflectivity images of the sample surface.

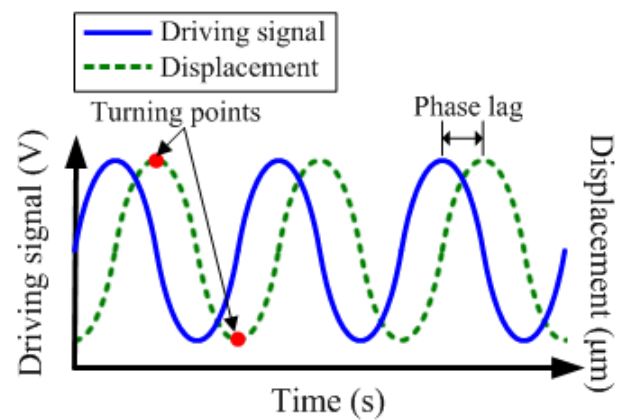

(a)

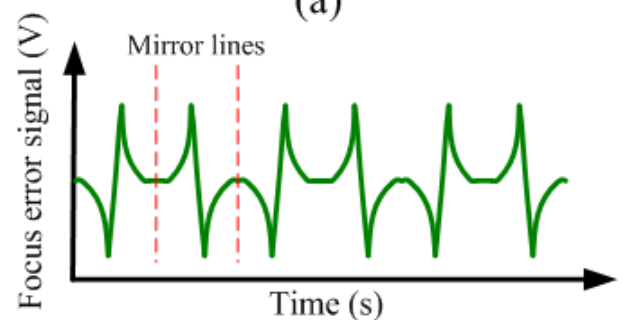

(b)

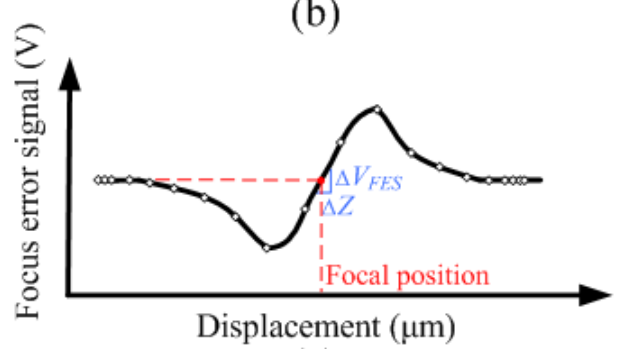

(c)

Fig. 4. Data analysis process for height calculation. (a) Driving signal and scanner displacement versus time, (b) $V_{F E S}$ versus time, and (c) $V_{F E S}$ versus scanner displacement.

\section{RESUlTS AND DisCUSSION}

In this section, the static and dynamic properties of the resonant scanner are described. A standard sample was measured using a z-axis modulation mode to obtain the images of quantitative height and reflectivity.

\section{A. Static displacement of the resonant scanner}

First, the static displacement of the resonant scanner was verified. A mirror was placed on the closed-loop XYZ scanner as a measured sample. The z-axis position of the mirror was adjusted to the focal position of the astigmatic pickup head by employing the approaching stage. The static driving voltage for the resonant scanner was varied from 0 to $150 \mathrm{~V}$ with an interval of $15 \mathrm{~V}$. When the driving voltage changed, the $\mathrm{Z}$-axis position of the closed-loop XYZ scanner was adjusted to maintain $V_{F E S}$ at the focal position. Therefore, the $\mathrm{Z}$-axis displacement of the closed-loop XYZ scanner was equal to the displacement of the resonant scanner. The relation between the displacement and driving voltage is shown in Fig. 5, which displays a typical hysteresis loop of the piezoelectric actuators. The maximum displacement was $7.4 \mu \mathrm{m}$ at a driving voltage of $150 \mathrm{~V}$.

\section{B. Dynamic property of the resonant scanner}

To verify the dynamic response of the resonant scanner, the same experimental setup with the static displacement measurement was used. The resonant scanner was operated using a sinusoidal waveform instead of a static voltage. To determine the resonant frequency of the scanner, the driving frequency was tuned manually with a constant amplitude of

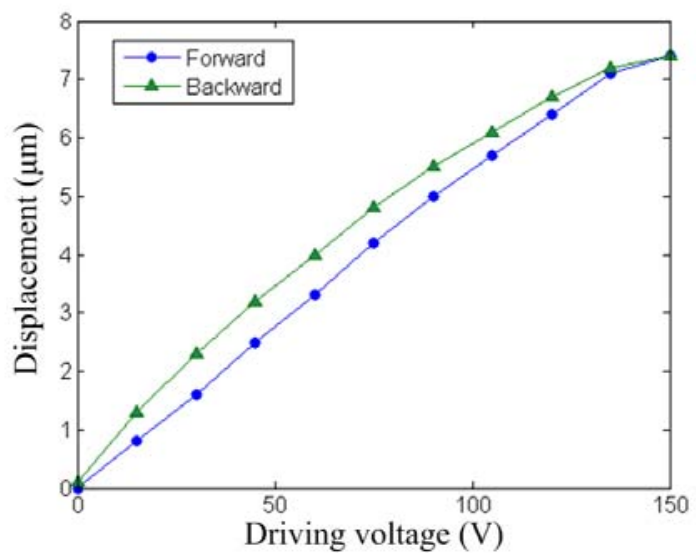

Fig. 5. Scanner displacement versus driving voltage.

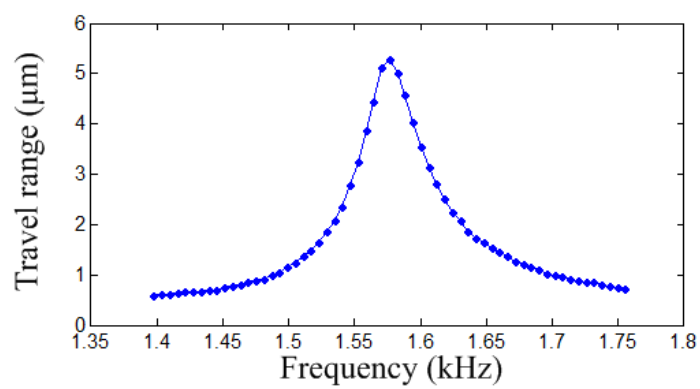

(a)

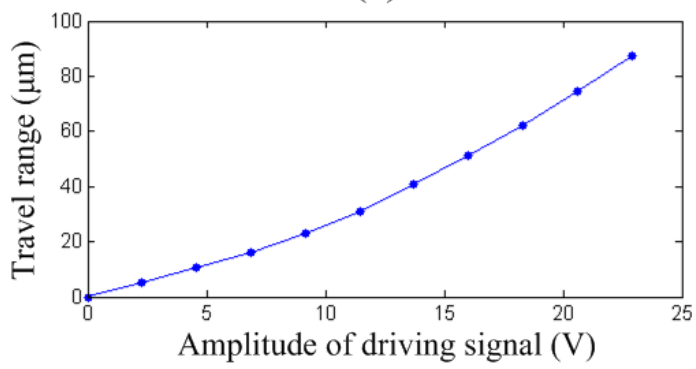

(b)

Fig. 6. (a) Travel range versus driving signal frequency with a driving amplitude of 2.25 V. (b) Travel range versus driving signal amplitude at 1.576 $\mathrm{kHz}$. 
$2.25 \mathrm{~V}$, the mirror sample was adjusted to the focal position, and $V_{F E S}$ was used to measure the scanner displacement. The amplitude of $V_{\text {FES }}$ was obtained using an oscilloscope, and converted to displacement by multiplying the reciprocal slope $\Delta Z / \Delta V_{F E S}$ of the linear region in the S-curve. The oscillation spectrum is presented in Fig. 6(a). The resonant peak had a quality factor of 36.6. The resonant frequency of the scanner was approximately $1.576 \mathrm{kHz}$, which was slightly lower than the resonant frequency of $1.94 \mathrm{kHz}$ obtained from simulation results. The error may have been caused by the neglected mass of the objective lens and polycarbonate chip. Moreover, the estimated density and Young's modulus in the simulation may not be identical to the actual material properties. To calibrate the travel range of the scanner, the distance between the two turning positions was measured using the closed-loop XYZ scanner. Figure 6(b) illustrates the relation between the travel range and driving amplitude at the resonant frequency of 1.576 $\mathrm{kHz}$. The travel range was positively proportional to the driving amplitude. A travel range of $87.3 \mu \mathrm{m}(43.65 \mu \mathrm{m}$ displacement amplitude) was achieved using a driving amplitude of $22.89 \mathrm{~V}$. This result indicated that the resonant mode can effectively magnify the travel range under a low driving amplitude.

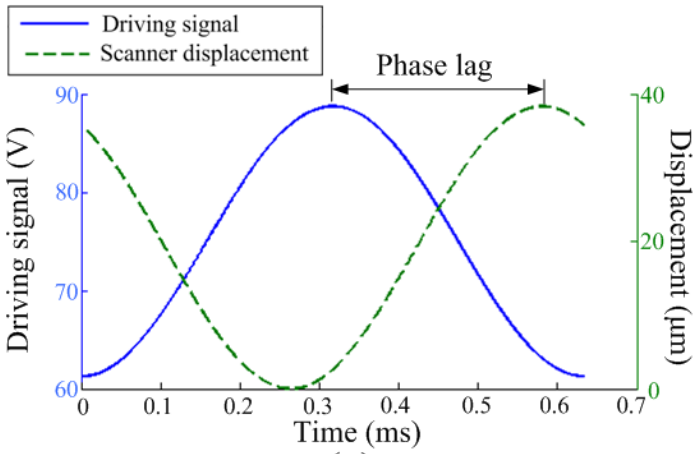

(a)

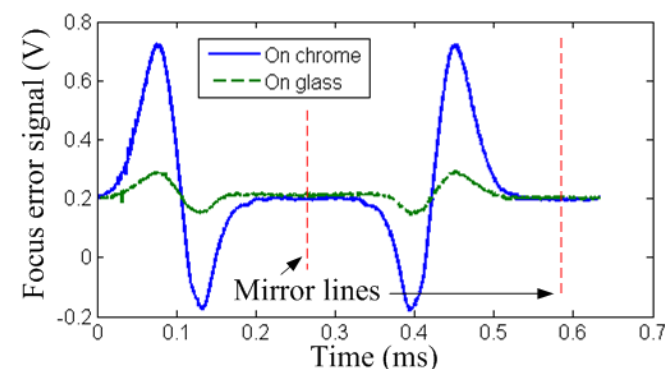

(b)

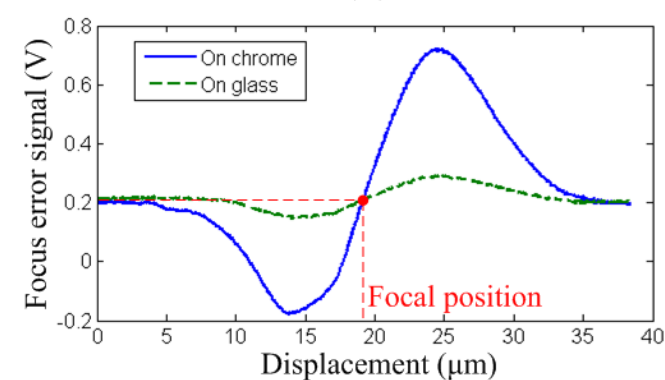

(c)

Fig. 7. (a) Driving signal and scanner displacement versus time. (b) $V_{F F S}$ versus time. (c) $V_{F E S}$ versus scanner displacement.

\section{Quantitative height and reflectivity imaging}

To examine the performance of an integrated optical profilometer, a standard sample (R2L2S1N1, ThorLab) was measured using the z-axis modulation mode. In this sample, a patterned chrome layer was coated on a glass substrate. The specification indicated that the thickness of the chrome layer was approximately $120 \mathrm{~nm}$. First, the sample was adjusted to the focal position of the pickup head, and then the xy position of the sample was manipulated to measure a region with line patterns. The resonant scanner was oscillated through the sinusoidal signal with an amplitude of $13.7 \mathrm{~V}$, and the corresponding travel range was $38.3 \mu \mathrm{m}$. The solid blue and green dashed lines [Fig. 7(a)] represent the driving signal and scanner displacement, respectively. A phase lag of $150^{\circ}(0.26$ $\mathrm{ms}$ time delay) existed between the driving signal and displacement of the scanner, which was obtained from the plot of $V_{F E S}$ vs. time as illustrated in Fig. 7(b). The solid blue and green dashed lines in Fig. 7(b) represent $V_{F E S}$ measured on the chrome and glass surfaces, respectively. Mirror lines indicate the turning points of the scanner displacement, which were employed to calibrate the phase lag and displacement amplitude. The focus error signal was captured at a constant sampling rate of $40 \mathrm{MHz}$. Therefore, a trade-off between the spatial resolution and travel range in the $\mathrm{z}$-axis direction existed. By using Fig. 7(a) and (b), the relation between $V_{F E S}$ and the scanner displacement was obtained as presented in Fig. 7(c). A peak-to-peak value on the chrome surface was higher than that on the glass surface, which was relative to the surface reflectivity.

To obtain the surface height and reflectivity images, the R2L2S1N1 sample was scanned in an area of $100 \mu \mathrm{m} \times 100 \mu \mathrm{m}$ with a resolution of 256 pixels $\times 256$ pixels. The scan and corresponding imaging rates were 1 line/s and $256 \mathrm{~s} /$ frame, respectively. The focal position and slope on each pixel were calculated to obtain the height and slope images, as presented in Fig. 8(a) and (b), respectively. The height image indicated that the average thickness of the chrome layer was $129.8 \pm 52.2 \mathrm{~nm}$ (mean \pm STD), which agreed with the previous result of $126 \pm$ $18 \mathrm{~nm}$ [12]. The large standard deviation was due to the rough chrome surface as shown in the height profile. Similar with the previous result, an image artifact occurred on the edges of chrome patterns, which caused artificial peaks and notches on the left and right edges, respectively. We speculated that the step geometry reflected the laser spot partially back to the PDIC, which caused an unbalanced laser power distribution on the PDIC segments and shifted the focus error signal. The average slopes on the chrome layer and glass substrate were $0.141 \pm$ 0.002 and $0.021 \pm 0.001 \mathrm{mV} / \mathrm{nm}$, respectively. The result confirmed that the reflectivity of the chrome surface was higher than that of the transparent glass. On comparing the height image with the slope image, the height image revealed small defects on the surface such as particles and scratches, which were not observed in the slope image. This result indicated that the analysis method can clearly decouple the surface height and reflectivity. 

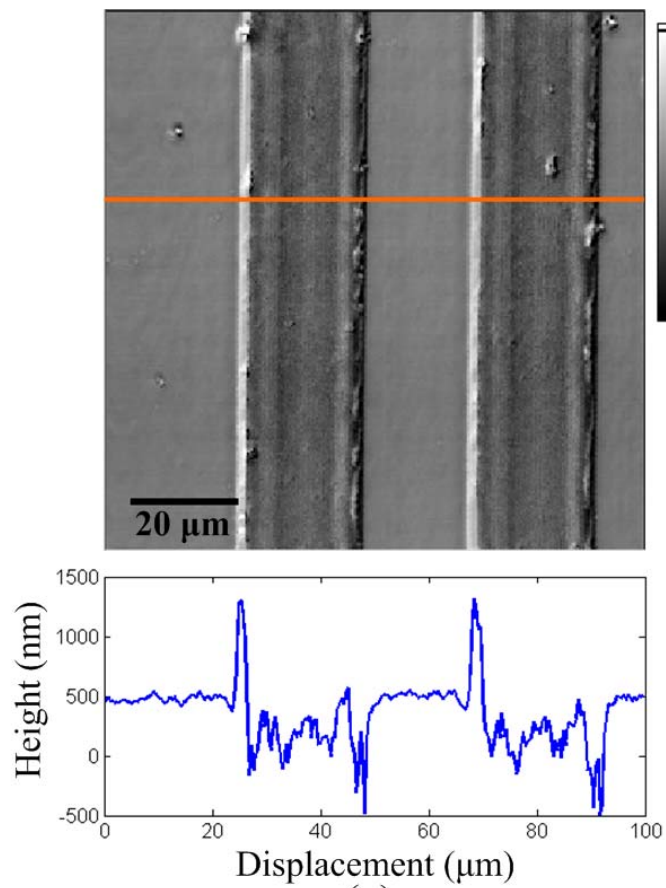

(a)
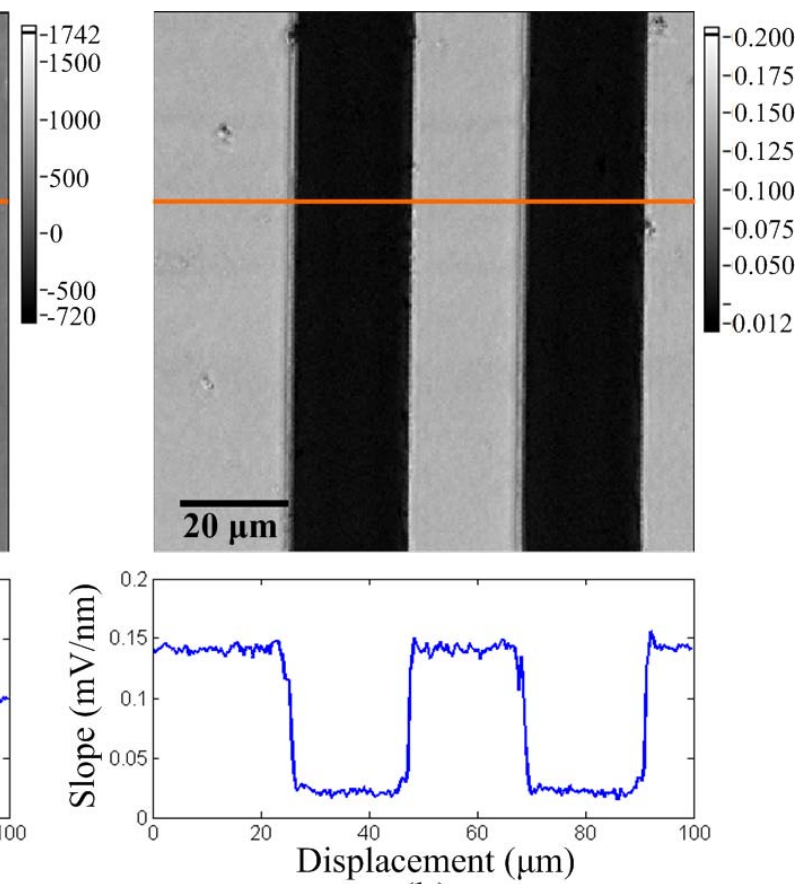

(b)

Fig. 8. (a) Height image and (b) slope image of the sample R2L2S1N1.

\section{Thermal effect of the resonant scanner}

A long time operation of the piezoelectric actuators at high speed may cause thermal expansion and a displacement drift. To test the thermal effect of the resonant scanner, $V_{F E S}$ was measured on the mirror sample to examine the distance variation between the resonant scanner and the sample surface. The mirror surface was first adjusted to the focal position and kept stationary during the measurement. Then, the resonant scanner was driven by a sinusoidal signal with a specified amplitude continuously, and $V_{F E S}$ was recorded every minute. To obtain static $V_{F E S}$ without the oscillation, the driving signal was temporarily set to $0 \mathrm{~V}$ in few seconds when $V_{F E S}$ was recorded. The relative displacement between the sample surface and the focal position was obtained through multiplying $V_{F E S}$ by the reciprocal slope $\Delta Z / \Delta V_{F E S}$. Figure 9 shows the displacement variations with different driving amplitudes of 0 , 6.9, and $13.7 \mathrm{~V}$. A slow fluctuation was observed in the displacement curve with $0 \mathrm{~V}$ driving amplitude, which may be caused by the environmental temperature variation. On the other hand, the displacement curves with driving amplitudes of 6.9 and $13.7 \mathrm{~V}$ rapidly increased to 75 and $200 \mathrm{~nm}$, respectively. We concluded that the rapid increase of the displacement was caused by the thermal expansion of the piezoelectric actuators, which moved the objective lens downward and generated a relative upward displacement of the sample surface to the focal position. Both displacement curves approached stable after 15 min approximately, because a thermal balance was achieved. At $19 \mathrm{~min}$, the driving amplitudes were set to $0 \mathrm{~V}$ again, and the displacement curves rapidly reduced back to the positions close to the focal position due to the thermal shrinkage.

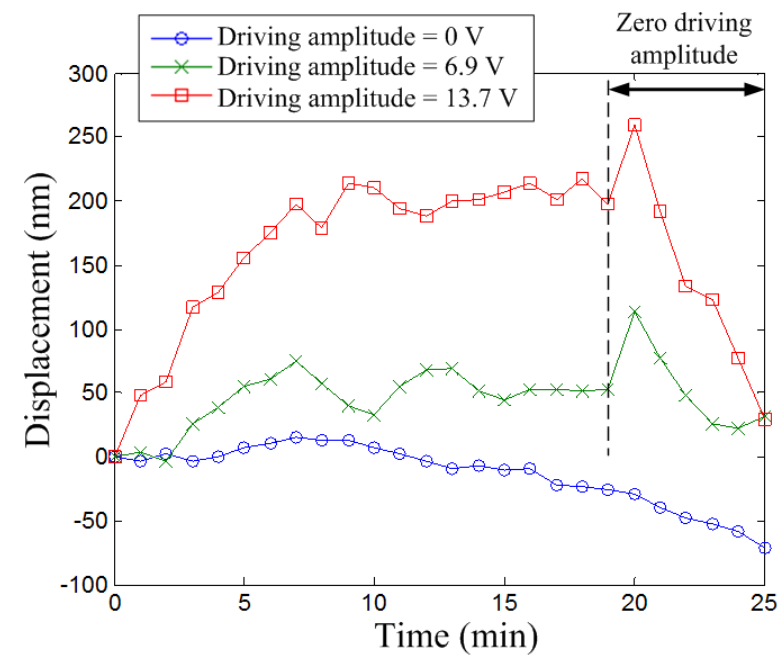

Fig. 9. Displacement versus time with different driving amplitudes.

\section{CONCLUSION}

In this work, a resonant scanner was proposed to increase the imaging rate of the z-axis modulation mode of the astigmatic optical profilometer. The resonant scanner was operated at its resonant frequency of approximately $1.576 \mathrm{kHz}$, which was approximately 130 times higher than the $\mathrm{z}$-modulation rate of $12 \mathrm{~Hz}$ using the original closed-loop XYZ scanner [12]. Moreover, the current imaging rate $(256 \mathrm{~s} /$ frame $)$ was approximately 50 times faster than the previous result $(3.5$ $\mathrm{h}$ /frame). An ideal image rate of $83 \mathrm{~s} /$ frame can be calculated by dividing the image pixel number by the z-modulation rate. However, the current scan rate was limited by the bandwidth of the closed-loop XYZ scanner in xy scanning. Furthermore, the 
resonant mode can highly magnify the travel range of the scanner. The experimental result demonstrated that a travel range of $87.3 \mu \mathrm{m}$ can be achieved by providing a driving amplitude of $22.89 \mathrm{~V}$. By contrast, a disadvantage of the resonant scanner was the nonlinear movement, which increased the complexity of the data analysis. We introduced a calibration procedure to reconstruct the $V_{F E S}$ versus displacement graph. The results verified that the analysis method can successfully decouple the surface height and reflectivity and provide the quantitative height image. Moreover, a continuous operation of the resonant scanner at high speed may cause thermal expansion and a drift on the height measurement. However, the experimental results demonstrated that a thermal balance can be achieved within 15 min at room temperature.

\section{ACKNOWLEDGMENT}

This research was financially supported by the Ministry of Science and Technology, Taiwan (MOST 108-2221-E-002-151, MOST 109-2221-E-002-086).

\section{REFERENCES}

[1] J. Tong, C. A. Simmons, and Y. Sun, "Precision patterning of PDMS membranes and applications," Journal of Micromechanics and Microengineering, vol. 18, no. 3 , 2008, Art. no. 037004.

[2] J. Szewczenko, J. Jaglarz, M. Basiaga, J. Kurzyk, and Z. Paszenda, "Optical methods applied in thickness and topography testing of passive layers on implantable titanium alloys," Optica Applicata, vol. 43, no. 1, pp. 173-180, 2013

[3] S. Haldal, Shashikala, P. Rajesh, and S. S. Ali, "Quantitative assessment of the surface roughness of two esthetic restorative materials after tooth brush abrasion using 3-D profilometer and scanning electron microscope," Int. J. Health Sci. Res., vol. 3, pp. 43-49, 2013.

[4] I. Sherrington and E. H. Smith, "Modern measurement techniques in surface metrology: part II; optical instruments," Wear, vol.125, no. 3, pp. 289-308, 1988

[5] R. J. Hocken, N. Chakraborty, and C. Brown, "Optical Metrology of Surfaces," CIRP Annals, vol. 54, no. 2, pp. 169-183, 2005.

[6] L.-C. Chen, Y.-T. Huang, and K.-C. Fan, "A Dynamic 3-D Surface Profilometer With Nanoscale Measurement Resolution and $\mathrm{MHz}$ Bandwidth for MEMS Characterization," IEEE/ASME Transactions on Mechatronics, vol. 12, no. 3, pp. 299-307, 2007.

[7] K.-I. Joo, M. Kim, M.-K. Park, H. Park, B. Kim, J. Hahn, and H.-R. Kim, "A 3D Optical Surface Profilometer Using a Dual-Frequency Liquid Crystal-Based Dynamic Fringe Pattern Generator," Sensors, vol. 16, no. 11 , pp. 1794, 2016

[8] K. Ehrmann, A. Ho, and K. Schindhelm, "A 3D optical profilometer using a compact disc reading head," Measurement Science and Technology, vol. 9, no. 8, pp. $1259-1265,1998$.

[9] E. T. Hwu, S. K. Hung, C. W. Yang, K. Y. Huang, and I. S. Hwang, "Real-time detection of linear and angular displacements with a modified DVD optical head," Nanotechnology, vol. 19, no. 11, 2008, Art. no. 115501.

[10]E. E.-T. Hwu, and A. Boisen, "Hacking CD/DVD/Blu-ray for Biosensing," ACS Sensors, vol. 3, no. 7, pp. 1222-1232, 2018.

[11]E. T. Hwu, H. Illers, L. Jusko, and H. U. Danzebrink, "A hybrid scanning probe microscope (SPM) module based on a DVD optical head," Measurement Science and Technology, vol. 20, no. 8, 2009, Art. no. 084005.

[12]H.-S. Liao, G.-T. Huang, H.-D. Tu, T.-H. Lin, and E.-T. Hwu, “A novel method for quantitative height measurement based on an astigmatic optical profilometer," Measurement Science and Technology, vol. 29, no. 10, 2018 , Art. no. 107002

[13] C. D. Near, "Piezoelectric actuator technology," in Proc. SPIE 2717, Smart Structures and Materials 1996: Smart Structures and Integrated Systems, vol. 2717, pp. 246-258, 1996.

[14] K. Uchino, "Piezoelectric actuators 2006," Journal of Electroceramics, vol. 20, no. 3-4, pp. 301-311, 2007.
[15]H. P. Syahputra, T. J. Ko, and B. M. Chung, "Development of 2-axis hybrid positioning system for precision contouring on micro-milling operation," Journal of Mechanical Science and Technology, vol. 28, no. 2, pp. 691-697, 2014

[16]O. M. E. Rifai and K. Youcef-Toumi, "Design and control of atomic force microscopes," in Proceedings of the 2003 American Control Conference, 2003, vol. 5, pp. 3714-3719, 2003.

[17] G. Schitter, K. J. Astrom, B. E. DeMartini, P. J. Thurner, K. L. Turner, and P. K. Hansma, "Design and Modeling of a High-Speed AFM-Scanner," IEEE Transactions on Control Systems Technology, vol. 15, no. 5, pp. 906-915, 2007.

[18]T. Ando, "High-speed atomic force microscopy coming of age," Nanotechnology, vol. 23, no. 6, 2012, Art. no. 062001.

[19] Y. K. Yong, S. O. Moheimani, B. J. Kenton, and K. K. Leang, "Invited review article: high-speed flexure-guided nanopositioning: mechanical design and control issues," Rev Sci Instrum, vol. 83, no. 12, 2012, Art. no. 121101.

[20]T. Tuma, W. Haeberle, H. Rothuizen, J. Lygeros, A. Pantazi, and A. Sebastian, "Dual-Stage Nanopositioning for High-Speed Scanning Probe Microscopy," IEEE/ASME Transactions on Mechatronics, vol. 19, no. 3, pp. 1035-1045, 2014.

[21] A. D. L. Humphris, M. J. Miles, and J. K. Hobbs, "A mechanical microscope: High-speed atomic force microscopy," Applied Physics Letters, vol. 86, no. 3, 2005, Art. no. 034106.

[22]B. Zhao, J. P. Howard-Knight, A. D. L. Humphris, L. Kailas, E. C. Ratcliffe, S. J. Foster, and J. K. Hobbs, "Large scan area high-speed atomic force microscopy using a resonant scanner," Review of Scientific Instruments, vol. 80, no. 9, 2009, Art. no. 093707.

[23]J. Zhao, W. Gong, W. Cai, and G. Shang, "Piezoelectric bimorph-based scanner in the tip-scan mode for high speed atomic force microscope," Review of Scientific Instruments, vol. 84, no. 8, 2013, Art. no. 083706.

[24] W. Cai, J. Zhao, W. Gong, H. Fan, and G. Shang, "Resonance-type bimorph-based high-speed atomic force microscopy: real-time imaging and distortion correction," Measurement Science and Technology, vol. 25, no. 12,2014 , Art. no. 125404.

[25]J. Y. Wang, N. Mullin, and J. K. Hobbs, "High-speed large area atomic force microscopy using a quartz resonator," Nanotechnology, vol. 29, no. 33, 2018, Art. no. 335502 . 